\title{
The Effect of Radiation on Free Convection from a Heated Horizontal Circular Cylinder
}

\author{
Najahulfazliah Zainuddin ${ }^{1}$, Ishak Hashim ${ }^{2}$, Muhaimin Ismoen ${ }^{1}$ \\ \& Rozaini Roslan ${ }^{1}$ \\ ${ }^{1}$ Centre for Research in Computational Mathematics \\ Faculty of Science, Technology and Human Development \\ Universiti Tun Hussein Onn \\ 86400 Batu Pahat, Johor DT, Malaysia \\ ${ }^{2}$ Centre for Modelling \& Data Analysis, School of Mathematical Sciences \\ Universiti Kebangsaan Malaysia \\ 43600 UKM Bangi, Selangor DE, Malaysia
}

Keywords: Radiation; free convection; heated circular cylinder.

\begin{abstract}
Effect of radiation on free convection on heated horizontal circular has been investigated. The cylinder is fixed and immersed in a stationary fluid, in which the temperature is uniformly heated about the temperature of the surrounding fluid. The governing equation are transformed into dimensionless non-linear of partial differential equations and solved numerically by employing a finite difference method. An implicit finite difference scheme of Crank Nicolson method is used to analyze the results. This study determine the effects of radiation parameter, $R_{d}$ heat generation parameter, $\gamma$ and the Prandtl number, $P r$, on the temperature and velocity profiles. The results of the local heat transfer and skin-friction coefficient in the presence of radiation for some selected values of Prandtl number and heat generation parameter have shown graphically.
\end{abstract}

\section{INTRODUCTION}

The effects of radiation on free convection boundary layer over a various shapes such as plate, sphere, cylinder and others have been studied among researchers because it has become more important in recent years. The reason is the thermal radiation effects on free convection flow are important in engineering applications, such as in advanced types of power plants for nuclear rockets, high-speed flights, re-entry vehicles and processes involving high temperature. The effect of heat generation in convection is also important and significant where there exist difference temperature between the surface and the ambient fluid. In certain applications on dealing with heat generation may alters the temperature and velocity distribution.

As the presence of heat generation where there is the difference between the surface temperature and the ambient temperature, the radiation effects is also important. In the study on the effect of radiation on free convection was studied by Soundalgekar et al. (1960) which consider on free convection flow of a gas past a semi-infinite flat plate using the Cogley et al. (1968) equilibrium model. While, Hossain \& Takhar (1996) have analyzed the effects of radiation on free convection flow past a heated vertical plate. Later Tahmina \& Alim et al. (2008) extended from the previous studies on a sphere with uniform surface heat. Besides that, Yih (1999) studied on a truncated cone by following the Rosseland diffusion approximation which also used by Molla et al. (2009b) on investigate the radiation effect on natural convection over vertical wavy frustum of cone. Then, Molla et al. (2011) extended their previous study on a horizontal circular cylinder. The radiative heat fluxes can be approximated by the Rosseland diffusion approximation which has been employed among researchers. Very recently, Elbashbeshy et al. (2014) was considered on the effect of heat generation or absorption and thermal radiation on free convection flow and heat transfer over a truncated cone in the presence of pressure work. 
Furthermore, the study on free convection with the presence both of heat generation and radiation on natural convection flow over a various type of shape was investigated. The study on a sphere was investigated by Miraj \& Alim (2010). Later, Miraj et al. (2011) extended his previous work to investigate the effects of pressure work and radiation on their problem. While, Makinde (2012) study on the hydromagnetic mixed convection stagnation point flow towards a vertical plate embedded in a highly porous medium. Recently, Ali et al. (2013) investigate MHD free convection flow along vertical flat plate. While, Ferdousi et al. (2013) studied on a porous vertical plate in presence of heat generation.

Ganesan \& Rani (1998) presented a numerical solution for the transient natural convection flow over a vertical cylinder under the combined buoyancy effects of heat and mass transfer by employing an implicit finite-difference method that has been used by Rani \& Devaraj (2003) in studied about unsteady flow past a vertical cylinder with temperature oscillations. A finite difference method is also presented in Abd El-Nabyet al. (2004) work who studied on radiation effects on MHD unsteady free convection flow over vertical porous plate. Rani \& Kim (2008) are also demonstrate the CrankNicolson type of implicit finite- difference method in their study on the transient free convection flow over an isothermal vertical cylinder with temperature dependent viscosity. The above method is also used by Reddy \& Reddy (2009a) and Reddy \& Reddy (2009b) on the interaction of radiation and mass transfer effects on unsteady MHD free convection flow past a semi-infinite moving vertical cylinder. Recently, Ismail et al. (2012) demonstrated a finite difference solution of radiation on unsteady free convective magnetohydrodynamic flow past a vertical cylinder with heat and mass transfer. They solve their problem by an implicit finite difference scheme of Crank-Nicolson method which is demonstrated in Velusamy \& Garg (1992) work. Very recently, Udin \& Harmand (2013) employed the an unconditionally stable implicit finite difference scheme to solve their problems on unsteady natural convection heat transfer of nanofluid along a vertical plate embedded in porous medium.

There was another works that solved numerically by using explicit finite difference method that demonstrated in Carnahan et al. (1969) in studied about free convection on a heated plate. The method has been used by Udin \& Kumar (2010) in the problem of unsteady free convection in a fluid past an inclined plate immersed in a porous medium. Besides that, the method is also has been employed by Khan et al. (2012) in his studied on unsteady MHD free convection boundary- layer flow of a nanofluid along a stretching sheet with thermal radiation and viscous dissipation effects.

In the present study, it is considered to investigate the natural convection flow on a heated horizontal circular cylinder in the presence of heat generation and radiation by employing the Rosseland diffusion approximation. The governing equations of this study are transformed into dimensionless forms, which are solved numerically using a Crank-Nicolson type implicit finite-difference. The numerical results have been obtained in terms of local skin friction, rate of heat transfer, velocity profiles as well as temperature profiles for set of different radiation parameter, heat generation parameter and Prandtl number.

\section{FORMULATION OF PROBLEM}

Free convection flow from a heated horizontal circular cylinder in the presence of radiation is considered. The flow over the horizontal circular cylinder is assumed to be two-dimensional, laminar, unsteady and incompressible. The physical configuration is as shown in the Figure 1. 


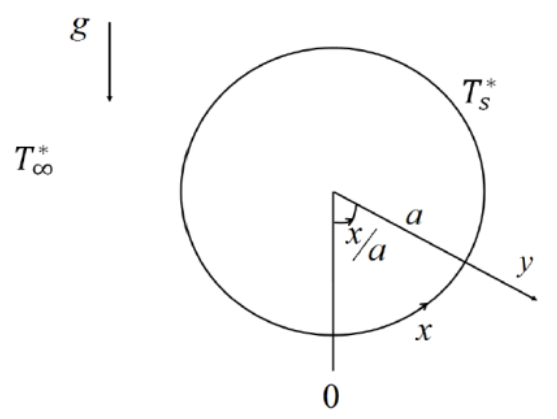

Figure 1: Physical model of circular cylinder

$T^{*}{ }_{S}$ is assumed as the surface temperature of the cylinder where $T^{*}{ }_{S}>T^{*}{ }_{\infty}$ and $T^{*}{ }_{\infty}$ is assumed as the ambient temperature of the fluid and $T^{*}$ is the temperature of the fluid. By extending the problem of free convection on heated plate by Carnahan et al. (1969) and the problem of the effect of on free convection over the circular cylinder from Molla et al. (2011) and Miraj \& Alim (2010), respectively are considered. By considering the present physical conditions, usual Boussinesq approximation and boundary layer approximations, the full of continuity, momentum and energy equations are formulated in the following forms:

$\frac{\partial u}{\partial x}+\frac{\partial v}{\partial y}=0$

$\frac{\partial u}{\partial t^{*}}+u \frac{\partial u}{\partial x}+v \frac{\partial u}{\partial y}=v\left(\frac{\partial^{2} u}{\partial y^{2}}\right)+g \beta\left(T^{*}-T_{\infty}^{*}\right) \sin \left(\frac{x}{a}\right)$

$\frac{\partial T^{*}}{\partial t^{*}}+u \frac{\partial T^{*}}{\partial x}+v \frac{\partial T^{*}}{\partial y}=\frac{\kappa}{\rho C_{\rho}}\left(\frac{\partial^{2} T^{*}}{\partial y^{2}}\right)-\frac{1}{\rho C_{\rho}}\left(\frac{\partial q_{r}^{\prime \prime}}{\partial y}\right)$

where the $x$-coordinate is defined as a distance measured along the surface of a circular cylinder in vertically upward direction and $y$-coordinate is the outwards a distance measured normally from the surface of the cylinder. $u$ and $v$ denote the velocity components in the $x$ and $y$ directions, respectively. The gravitational acceleration $g$ is acting downward and $\beta$ is the coefficient of thermal expansion, $(x / a)$ is the angle of the $y$-axis, $T^{*}$ is the temperature of fluid and $T^{*}{ }_{\infty}$ is the temperature away from the cylinder, $\kappa$ is the thermal conductivity, $\rho$ is the fluid density, $C_{\rho}$ is the specific heat of constant pressure, and $q_{r}^{\prime \prime}$ is represents the radiative heat flux in the $y$ direction. The boundary conditions are taken as

$u=v=0, T^{*}=T^{*}$ at $y=0$,

$u \rightarrow 0, v \rightarrow 0, T^{*} \rightarrow T_{\infty}^{*}$ as $y \rightarrow \infty$.

In equation (3), the term $q_{r}^{\prime \prime}$ the radiative heat flux which is as Rosseland diffusion approximation obtained by Rosseland (1936) and is given by

$q_{r}^{\prime \prime}=\frac{4 \sigma}{3 \kappa\left(\alpha_{r}+\sigma_{s}\right)} \frac{\partial T^{* 4}}{\partial y}$

Where $\sigma$ the Stefan-Boltzmann constant, $\alpha_{r}$ is Rosseland mean absorption coefficient and $\sigma_{s}$ is the scattering coefficient. The above expression that measures the radiative heat transfer, $q_{r}^{\prime \prime}$ is appropriate 
for two-dimensional boundary later flow. In order to non-dimensionalize equations. (1)-(4) the following dimensionless variables are introduced:

$X=\frac{x}{a}, \quad Y=\frac{y}{a} G r^{1 / 4}, \quad t=\frac{a^{2}}{v} G r^{-1 / 2} t^{*}, \quad U=\frac{a}{v} G r^{1 / 2} u, \quad V=\frac{a}{v} G r^{-1 / 4} v$,

$T=\frac{T^{*}-T_{\infty}^{*}}{T^{*}{ }_{s}-T^{*}{ }_{\infty}}, G r=g \beta \frac{\left(T^{*}{ }_{s}-T^{*}{ }_{\infty}\right)}{v^{2}} a^{3}$,

$T_{s}=\frac{T^{*}{ }_{s}}{T_{\infty}^{*}}, \quad \Delta=T_{s}-1, \quad R_{d}=\frac{4 \sigma T_{\infty}^{* 3}}{\kappa\left(\alpha_{r}+\sigma_{s}\right)}, \quad v=\frac{\mu}{\rho}, \quad \operatorname{Pr}=\frac{\mu C_{\rho}}{k}$,

where $v$ is the reference kinematic viscosity, $G r$ is the Grashof number, $T$ is non-dimensional temperature function, $T_{s}$ is the surface temperature parameter, $R_{d}$ is radiation parameter, $\operatorname{Pr}$ is the Prandtl number. Convert the governing equations (1)-(3) and the boundary conditions (4) into dimensional forms by substituting the non-dimensional variables above into the equations (1) - (4) and becomes to the following form:

$\frac{\partial U}{\partial X}+\frac{\partial V}{\partial Y}=0$

$\frac{\partial U}{\partial t}+U \frac{\partial U}{\partial X}+V \frac{\partial U}{\partial Y}=\frac{\partial^{2} U}{\partial Y^{2}}+T \sin X$

$\frac{\partial T}{\partial t}+U \frac{\partial T}{\partial X}+V \frac{\partial T}{\partial Y}=\frac{1}{P r} \frac{\partial}{\partial Y^{2}}\left[\left\{1+\frac{4}{3} R_{d}(1+\Delta T)^{3}\right\} \frac{\partial T}{\partial Y}\right]$.

The corresponding boundary conditions in dimensionless form are as follows:

$U=V=0, T=1$ at $Y=0$,

$U \rightarrow 0, V \rightarrow 0, T \rightarrow 0$ as $Y \rightarrow \infty$.

The physical quantities of principle interest are the rate of heat transfer and the shearing stress, $\tau_{s}$ in terms of the Nusselt number, $N u$ and skin-friction coefficient, $C_{f}$ respectively, which can be written as

$N u=\frac{a\left(q_{s}^{\prime \prime}+q_{r}^{\prime \prime}\right)_{y=0}}{\kappa\left(T^{*}{ }_{s}-T^{*}{ }_{\infty}\right)}, \quad C_{f}=\frac{\left(\tau_{s}\right)_{y=0}}{\rho U_{\infty}^{2}}$

where $\tau_{s}=\mu(\partial u / \partial y)$ and $q_{s}^{\prime \prime}=-k\left(\partial T^{*} / \partial y\right)$. By using the non-dimensional variables (6) into equations (11) made the equation becomes in dimensionless forms:

$N u G r^{-\frac{1}{4}}=-\left.\left(1+\frac{4}{3} R_{d} T_{s}^{3}\right) \frac{\partial T}{\partial Y}\right|_{Y=0}$,

$C_{f} G r^{\frac{1}{4}}=\left.X \frac{\partial^{2} T}{\partial Y^{2}}\right|_{Y=0}$

An implicit finite difference scheme of Crank Nicolson type is employed to solve the non-linear partial differential equations (7)-(9) under the boundary conditions (10). The region of solution is considered from the beginning of stagnation point $X=0$ to the end stagnation point $X_{\max }=\pi$ and an 
axis normal to the surface from $Y=0$ to $Y_{\max }=30.0$ as corresponding to $Y_{\infty}$. The subscripts $i$ and $j$ designate the grid points along the $X$ and $Y$ coordinates, respectively where $X_{i}=i \Delta X$ and $Y_{j}=j \Delta Y$ and the subscript $k$ designates the value of time $t=k \Delta t$ with the mesh sizes $\Delta X=\pi / 150, \Delta Y=0.12$ with the time step size dependency of $\Delta t=0.01$. The iterative procedure is stopped when the time step reached to $t_{\max }=10$. The finite difference equations corresponding to equations (7)-(9) are as follows:

\section{Crank-Nicolson method}

The equations (7)-(9) subject to the boundary conditions (10) are discretized using Crank-Nicolson types of implicit finite difference method and finally the system of tri-diagonal system of equations are obtained. Such a system of equations solved by Thomas algorithm as described in Carnahan et al. (1969).

$$
\begin{aligned}
& \frac{1}{2}\left[\frac{V_{i, j}^{k+1}-V_{i, j-1}^{k+1}}{\Delta Y}+\frac{V_{i, j}^{k}-V_{i, j-1}^{k}}{\Delta Y}\right]=\frac{1}{2}\left[\frac{U_{i, j}^{k+1}-U_{i-1, j}^{k+1}}{\Delta X}+\frac{U_{i, j}^{k}-U_{i-1, j}^{k}}{\Delta X}\right], \\
& \begin{aligned}
\frac{U_{i, j}^{k+1}-U_{i, j}^{k}}{\Delta t}+ & \frac{1}{2}\left[U_{i, j}^{k}\left(\frac{U_{i, j}^{k+1}-U_{i-1, j}^{k+1}+U_{i, j}^{k}-U_{i-1, j}^{k}}{\Delta X}\right)+V_{i, j}^{k}\left(\frac{U_{i, j}^{k+1}-U_{i, j-1}^{k+1}+U_{i, j}^{k}-U_{i, j-1}^{k}}{\Delta Y}\right)\right] \\
& =\frac{1}{2}\left[\left(\frac{U_{i, j}^{k+1}-2 U_{i, j}^{k+1}+U_{i, j-1}^{k+1}+U_{i, j+1}^{k}-2 U_{i, j}^{k}+U_{i, j-1}^{k}}{\Delta Y^{2}}\right)\right. \\
& \left.-\left(T_{i, j}^{k+1}+T_{i, j}^{k}\right) \sin X_{i}\right],
\end{aligned}
\end{aligned}
$$

$$
\begin{aligned}
\frac{T_{i, j}^{k+1}-T_{i, j}^{k}}{\Delta t}= & \frac{1}{2}\left[-U_{i, j}^{k} \frac{T_{i+1, j}^{k+1}-T_{i-1, j}^{k+1}+T_{i+1, j}^{k}-T_{i-1, j}^{k}}{2 \Delta X}-V_{i, j}^{k} \frac{T_{i, j}^{k+1}-T_{i, j-1}^{k+1}+T_{i, j}^{k}-T_{i, j-1}^{k}}{\Delta Y}\right] \\
& +\frac{1}{2}\left[\frac{1}{\operatorname{Pr}} \frac{\partial}{\partial Y}\left[\left\{1+\frac{4}{3} R_{d}\left(1+\left(T_{S}-1\right) T_{i, j}^{k}\right)^{3}\right\}\left\{\frac{T_{i, j}^{k+1}-T_{i, j-1}^{k+1}+T_{i, j}^{k}-T_{i, j-1}^{k}}{\Delta Y}\right\}\right] .\right.
\end{aligned}
$$

\section{NUMERICAL RESULTS}

The numerical results of study on free convection on a heated horizontal circular cylinder in the presence of heat generation are presented. This work was considered the effect of radiation parameter and heat generation parameter on the rate of heat transfer, the skin-friction coefficient, the temperature and velocity profiles. The effect of different Prandtl number will also be presented. Here, the Prandtl number used are $\operatorname{Pr}=0.7,4.0,7.0$ and the effects of radiation parameter $R_{d}=0.0,0.2,0.5,1.0$. The numerical results of the local heat trasnfer or Nusselt number and the local skin-friction coefficient are obtained in Table 1 represented the comparisons between of the present study with the previous study Molla et al.(2011). Here, Prandtl numbers $\operatorname{Pr}=0.73$, has been chosen. It is observed from the table that the present result has a good agreement with the results of Molla et al. (2011).

The effect for different values of radiation parameter $R_{d}$ on the velocity and temperature profiles with $\operatorname{Pr}=0.7$ and surface temperature parameter $T_{s}=1.0$ are shown in Figures 1 and 2 . Here, as $R_{d}$ increase, both the velocity and the temperature increase because the thermal boundary layer thickness increases and the thickness of the velocity boundary layer also increase. Which means the velocity and temperature gradients at the surface increase enhances the fluid velocity and temperature. The local maximum of the velocity exist within the boundary layer, but velocity increases 
near the surface of the cylinder and then decrease slowly approaches to zero while, the temperature from the surface decreases slowly and finally tend to zero. The change of velocity and temperature profiles in the $Y$ direction satisfied the natural convection boundary layer flow.

TABLE 1. Comparison between the present results and the previous study by Molla et al. (2011) for the rate of heat transfer $\mathrm{NuGr}^{-1 / 4}$ nd the local skin friction coefficients $C_{f} G r^{1 / 4}$ when $T_{S}=1.1$, $R_{d}=0.5$ and $\operatorname{Pr}=0.73$.

\begin{tabular}{|c|c|c|c|c|}
\hline \multirow{2}{*}{$X$} & \multicolumn{2}{|c|}{$N u \mathrm{Gr}^{-1 / 4}$} & \multicolumn{2}{c|}{$C_{f} G r^{1 / 4}$} \\
\cline { 2 - 5 } & $\begin{array}{c}\text { Present } \\
\text { study }\end{array}$ & $\begin{array}{c}\text { Molla et al. } \\
(2011)\end{array}$ & $\begin{array}{c}\text { Present } \\
\text { study }\end{array}$ & $\begin{array}{c}\text { Molla et al. } \\
(2011)\end{array}$ \\
\hline 0 & 0.54492 & 0.54424 & 0.0000 & 0.0000 \\
\hline$\pi / 6$ & 0.53773 & 0.53776 & 0.46937 & 0.46890 \\
\hline$\pi / 3$ & 0.51852 & 0.51845 & 0.86103 & 0.85457 \\
\hline$\pi / 2$ & 0.48592 & 0.48592 & 1.07755 & 1.08646 \\
\hline $2 \pi / 3$ & 0.43871 & 0.43887 & 1.11688 & 1.11449 \\
\hline $5 \pi / 6$ & 0.37512 & 0.37391 & 0.92132 & 0.91093 \\
\hline$\pi$ & 0.27337 & 0.27466 & 0.44234 & 0.44387 \\
\hline
\end{tabular}

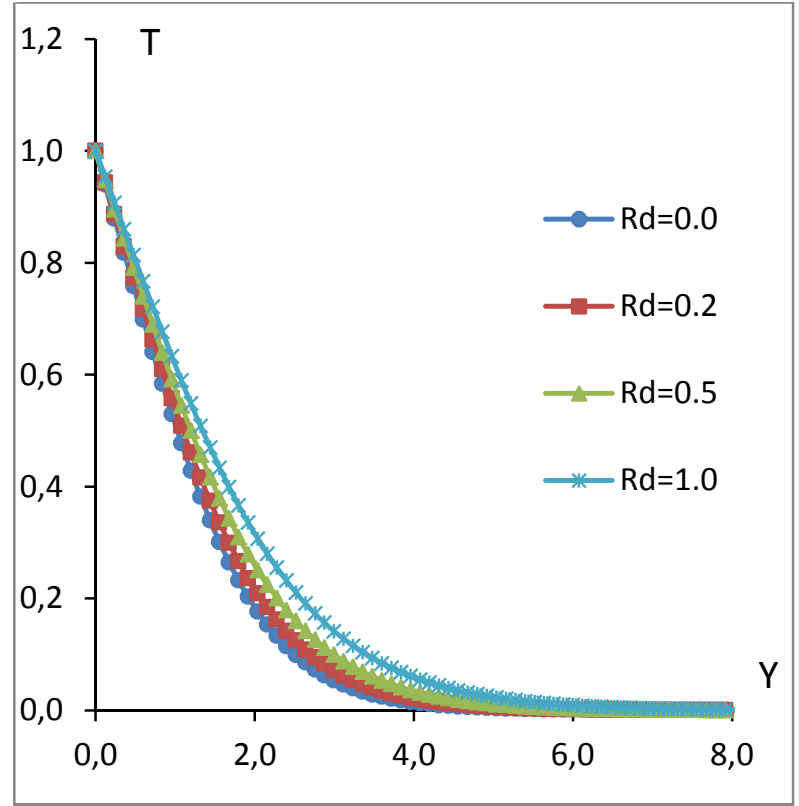

FIGURE 1. Temperature profile against $Y$ with FIGURE 2. Velocity profile against $Y$ with $\gamma=0.0$ and $\operatorname{Pr}=0.7$ at $t=4.0$ and $X=\pi / 6$.

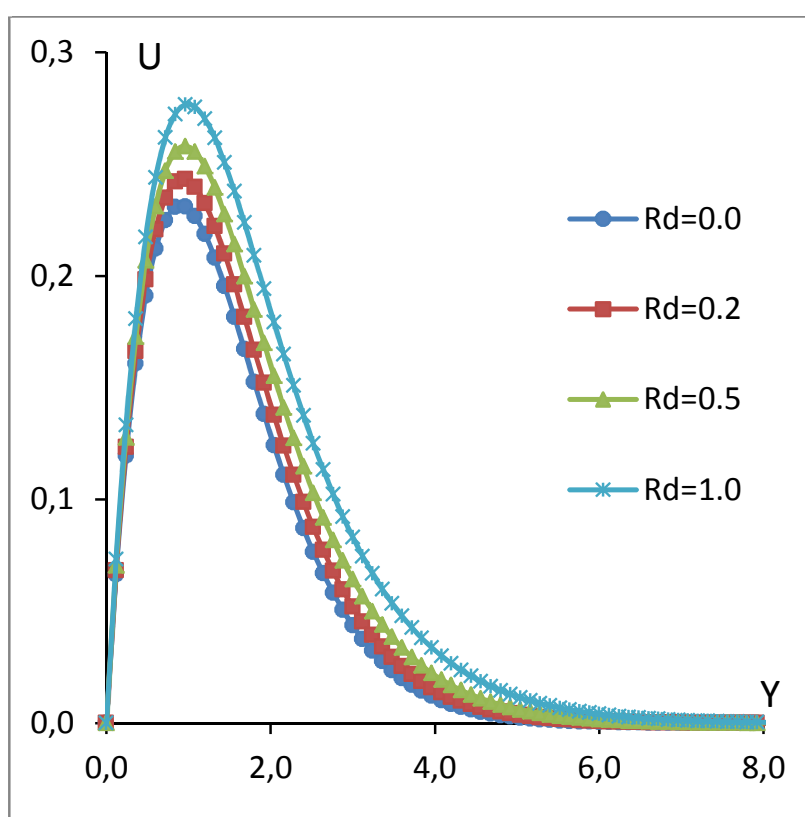

$\gamma=0.0$ and $\operatorname{Pr}=0.7$ at $t=4.0$ and $X=\pi / 6$.

Figures 3 and 4 show the local rate of heat transfer $N u$ and the local skin-friction coefficient $C_{f}$ respectively, increase for increasing values of $R_{d}$, while $\operatorname{Pr}=0.7$ and $t=4.0$. It is found that from the Figure 4, the skin friction increase gradually from zero value at beginning stagnation point $X=0$ along the $X$ direction and slowly decrease to downstream point $X_{\max }=\pi$. From Figure 3 , it reveals that the rate of heat transfer decrease slightly along the $X$ direction from beginning stagnation point to the downstream. 


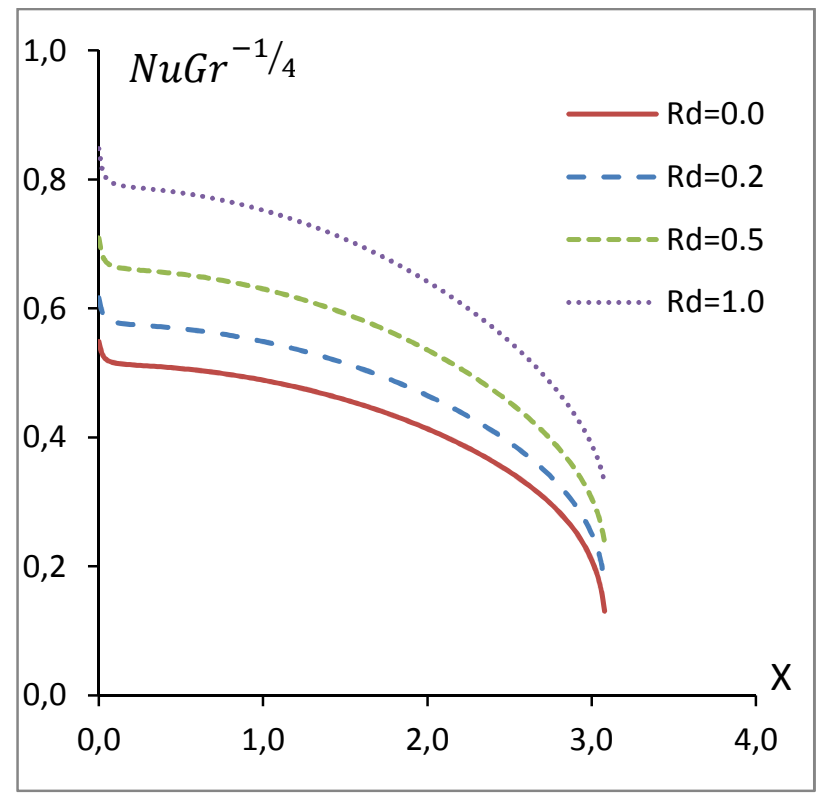

FIGURE 3. Local heat trasfer or Nusselt number with $\gamma=0.0$ and $\operatorname{Pr}=0.7$ at $t=4.0$.

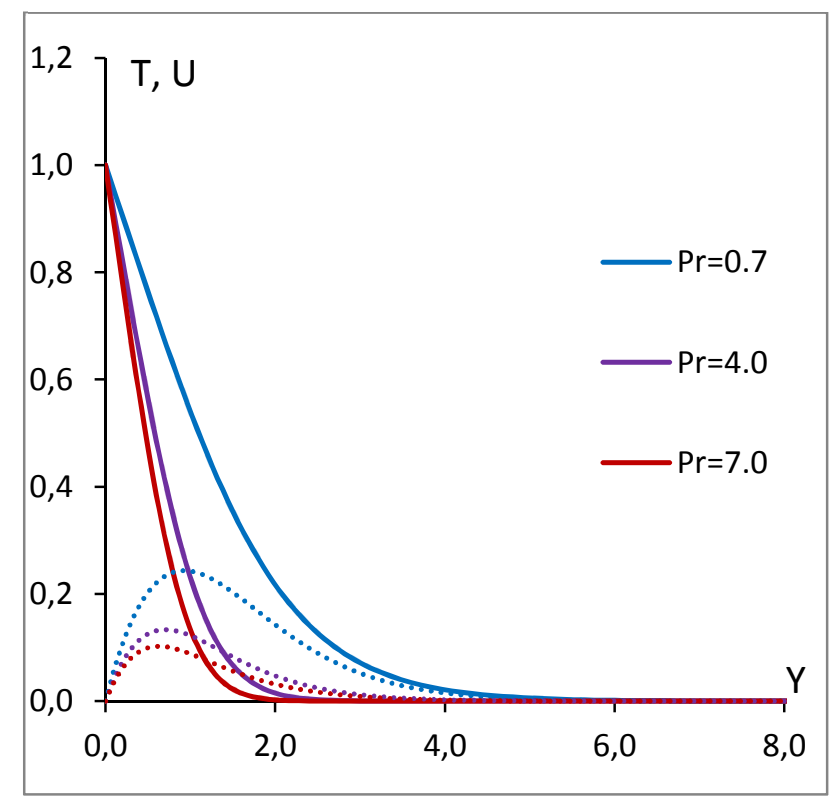

FIGURE 5. Temperature and velocity profiles against $Y$ with $R_{d}=0.2$ and $\operatorname{Pr}=0.7$ at $t=4.0$ and $X=\pi / 6$

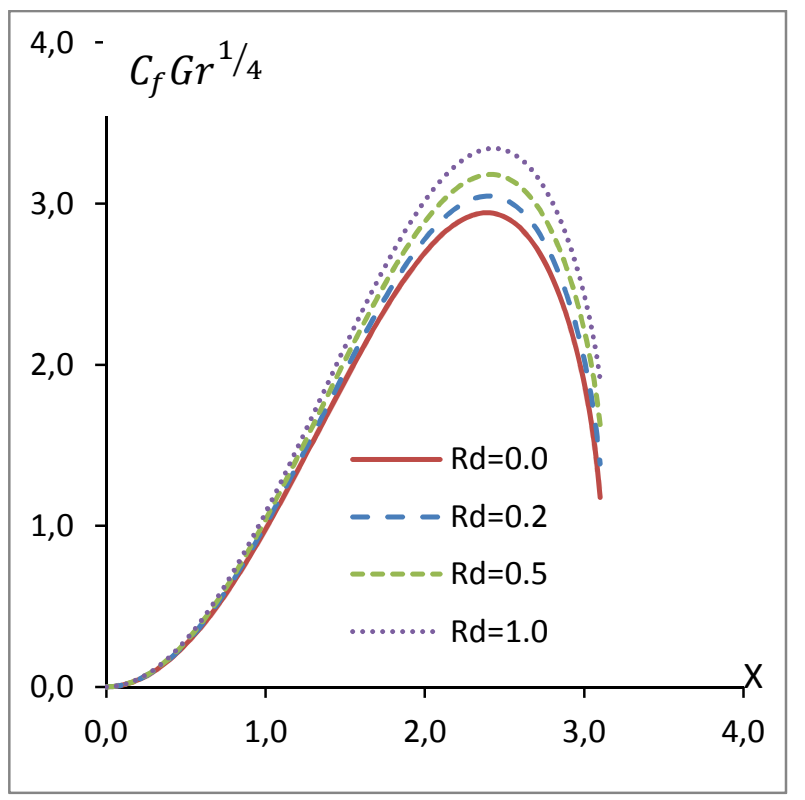

FIGURE 4. Local skin friction with $\gamma=0.0$ and $\operatorname{Pr}=0.7$ at $t=4.0$.

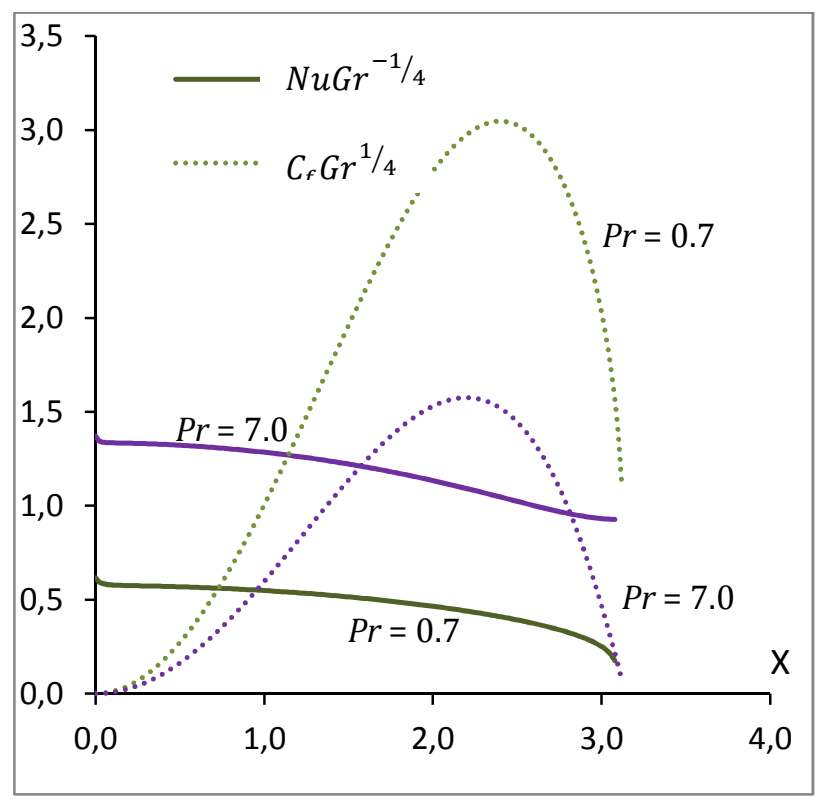

FIGURE 6. Local skin friction and Nusselt number with $\gamma=0.0$ and $R_{d}=0.2$ at $t=$ 4.0 and $X=\pi / 6$.

The variation of $\operatorname{Pr}$ on the velocity and temperature profiles when $R_{d}=0.2$ at $t=4.0$ are shown in the Figures 5. It is found that as the $\mathrm{Pr}$ increase, then both of the temperature and velocity decrease as well as the thermal boundary layer thickness decrease significantly. Variations of the local skin friction coefficient $C_{f}$ and local Nusselt number $N u$ for different values of $\operatorname{Pr}$ while $R_{d}=0.2$ at $t=4.0$ are displayed in the Figures 6. From the figures, as $\operatorname{Pr}$ increase, the skin friction coefficient decrease and heat transfer coefficient increase. 


\section{CONCLUSION}

The effect of radiation on natural convection flow on a heated horizontal cylinder has been investigated for different values of relevant physical parameters. From the results presented, the comparison between the present results and the previous study are found to be in good agreement. The results are also show an increase in the values of radiation parameter leads to increase in the velocity profile, the temperature profile, the local skin friction coefficient and the local rate of heat transfer. Besides that, the increase in Prandtl number leads to decrease in all the velocity profile, the temperature profile, the local skin friction coefficient but increase the local rate of heat transfer.

\section{ACKNOWLEDGMENTS}

The author would like to acknowledge the financial support received from University Tun Hussein Onn Malaysia (UTHM) (Fundamental Research Grant Scheme FRGS/2/2010/SG/UTHM/02/4/0761).

\section{REFERENCES}

[1] Abd El-Naby, M. A., Elsayed, M. E. E \& Nader, Y. A. (2003). Finite difference solution of radiation effects on MHD unsteady free convection flow over vertical porous plate. Applied Mathematics and Computation, (151), pp. 327-346.

[2] Ali, M. M., Mamun, A. A., Maleque, M. A. \& Azim, NHM. A. (2013). Radiation effects on MHD free convection flow along vertical flat plate in presence of Joule heating and heat generation. Proc. of the Fifth BSME International Conference on Thermal engineering, (56), pp. 503-509.

[3] Carnahan, B., Luther, H. A. \&Wilkes, J. O. (1969). Applied Numerical Methods. New York: Wiley.

[4] Elbashbeshy, E. M. A., Emam, T. G. \& Sayed, E. A. A. (2014). Effect of thermal radiation on free convection flow and heat transfer over a truncated cone in the presence of pressure work and heat generation/absorption. Thermal Science 2014, (00), pp. 6.

[5] Cogley, A. C., Vincenti W. G. \& Giles S. E. (1968). Differential Approximation for Radiation Transfer in a Non Gray Near Equilibrium. New York: Springer.

[6] Ferdousi, A., Rahman, M. M., Parvez, M. S. \& Alim, M. A. (2013). Effect of radiation on natural convection flow from a porous vertical plate in presence of heat generation. International Journal of Engineering and Applied Sciences, (3(4)) pp. 20-29.

[7] Ganesan, P. \& Rani, H. P. (1998). Transient natural convection flow over vertical cylinder with heat and mass transfer. Journal of Heat and Mass Transfer, (33), pp. 449-455.

[8] Hossain, M. A. \& Takhar, H. S. (1996). Radiation effect on mixed convection along a vertical plate with uniform surface temperature. Heat and Mass Transfer, (31), pp. 243248.

[9] Ismail, M. N., Mohamadien, G. F., \& Gker, . D. (2012). Finite difference solution of radiation on unsteady free convective magnetohydrodynamic flow past a vertical cylinder with heat and mass transfer. arXiv preprint arXiv. 1206.3045.

[10] Khan, M. S. Karim, I., Ali, L. E. \& Islam, A. (2012). Unsteady MHD free convection boundary-layer flow of a nanofluid along a stretching sheet with thermal radiation and viscous dissipation effects. International Nano Letters, (2(24)8), pp. 1-9.

[11] Miraj, M. \& Alim, M. A. (2010). Effect of radiation on natural convection flow on a sphere in presence of heat generation. International Communications in Heat and Mass Transfer, (37), pp. 660-665.

[12] Miraj, M., Alim, M. A. \& Andallah, L. S.(2011). Effects of pressure work and radiation on natural convection flow around a sphere with heat generation. International Communications in Heat and Mass Transfer, (38), pp. 911-916. 
[13] Makinde, O. D. (2012). Heat and mass transfer by MHD mixed convection stagnation point flow toward a vertical plate embedded in a highly porous medium with radiation and internal heat generation. Meccanica, (47), pp. 1173-1184.

[14] Molla, M. M., Hossain, M. A. \& Gorla, R. S. R. (2009b). Radiation effect on natural convection boundary layer flow over a vertical wavy frustum of cone. Applied Mathematic Mechanical Engineering Science, (223) pp. 1605-1614.

[15] Molla, M. M., Saha, S. C., Khan, M. A. I., \& Hossain, M. A. (2011). Radiation effects on natural convection laminar flow from a horizontal circular cylinder. Desalination and Water Treatment, (30), pp. 89-97.

[16] Rani, H. R. \& Devaraj, R. (2003). Numerical solution of unsteady flow past a vertical cylinder with temperature oscillations. Foshung in Ingenieurwesen, (68), pp. 75-78.

[17] Rani, H. R. \& Kim, C. N. (2008). Transient free convection flow over an isothermal vertical cylinder with temperature dependent viscousity. Korean Journal Chemical Engineering, (25(1)), pp. 34-40.

[18] Reddy, M. M. G. \& Reddy, N. B. (2009a). Radiation and mass transfer effects on unsteady MHD free convection flow of an incompressible viscous fluid past a moving vertical cylinder. Theoretical Applied Mechanics, (36(3)), pp. 239-260.

[19] Reddy, M.M.G. \& Reddy, N.B. (2009b). Thermal radiation and mass transfer effects on MHD free convection flow of an vertical cylinder with variable surface temperature and concentration. Journal of Naval Architecture and Marine Engineering, 6(1), pp. 1-24.

[20] Soundalgekar, V. M., Takhar H. S. \& Vighnesam N.V. (1960 ). The combined free and forced convection flow past a semi-infinite vertical plate with variable surface temperature. Nuclear Engineering and Design, (110), pp. 95-98.

[21] Tahmina, A. \& Alim, M.A. (2008). Effects of radiation on natural convection flow around a sphere with uniform surface heat flux. Journal Mechanical Enggineering, (39(1)), pp. 50-56.

[22] Udin, Z. \& Kumar, M. (2010). Unsteady free convection in a fluid past an inclined plate immersed in a porous medium. Computer Modelling and New Technologies, (14), pp. 41-47.

[23] Udin, Z. \& Harmand, S. (2013). Natural convection heat transfer of nanofluids along a vertical plate embedded in porous medium. Nanoscale Research Letters, (8), pp.64.

[24] Velusamy, K. \& Garg, V. K. (1992). Transient natural convection over a heat generating vertical cylinder. International Journal Heat Mass Transfer, (35), pp. 1293-1306.

[25] Yih, K. A. (1999). Effect of radiation on natural convection about a truncated cone. International Journal and Mass Transfer, (42), pp. 4299-4305. 